\title{
Feasibility, validity and acceptability of self-collected samples for human papillomavirus (HPV) testing in rural Malawi
}

\section{Allahna Esber ${ }^{1}$, Alison Norris ${ }^{1}$, Enock Jumbe ${ }^{2}$, Jonathan Kandodo ${ }^{2}$, Patrick Nampandeni ${ }^{2}$, Patricia Carr Reese ${ }^{3}$, Abigail Norris Turner ${ }^{4}$}

1. Division of Epidemiology, The Ohio State University, Columbus, USA

2. Child Legacy International, Umoyo wa Thanzi Research, Lilongwe Malawi

3. School of Medicine, The George Washington University, Washington, DC, USA

4. Division of Infectious Diseases, Department of Internal Medicine, The Ohio State University, Columbus, USA
Date Received: 22-August-2017

Revision Received: 19-Dec-2017

Date Accepted: 30-Jan-2018

Correspondence:

Allahna Esber

esber.8@osu.edu

https://dx.doi.org/10.4314/mmi.v30i2.2

\begin{abstract}
Aim
Abstract

The World Health Organization (WHO) recently endorsed human papillomavirus (HPV) testing as a cervical cancer screening method in countries without established programs. Self-collection for HPV testing may be an effective way to expand screening. Our objective was to assess the feasibility, validity, and acceptability of self-collection for HPV testing in a population of care-seeking, unscreened women in rural Malawi.
\end{abstract}

\section{Methods}

We enrolled women reporting to a rural Malawian clinic from January to August 2015. Participants were offered the option to selfcollect a vaginal sample and the study clinician collected a cervical sample for HPV testing. Using the clinician-collected sample as the reference standard, we calculated a kappa statistic, sensitivity, and specificity by hr-HPV type. Participants also received a brief survey assessing acceptability of the procedure.

\section{Results}

Among the 199 enrolled women, 22\% had any high risk-HPV. Comparing self- and clinician-collected samples for HPV testing, we found generally high agreement $(x=0.66-0.90)$ and high specificity $(98 \%-100 \%)$, but varied sensitivity $(50 \%-91 \%)$ for different types of hr-HPV. We also found that self-collection was acceptable, with $98 \%$ of women reporting it was easy to do and $99 \%$ reporting willingness to do so again.

\section{Conclusions}

WHO guidelines recommend that treatment is available immediately after a positive screening test for clinic-based cervical cancer screening programs. Our findings demonstrate that self-collection of samples for HPV testing is a feasible and acceptable method of cervical cancer screening in this rural Malawian population. High agreement between the self- and clinician-collected samples and high levels of acceptability among women in the study suggest that self-collection of vaginal samples for HPV testing may be effectively incorporated into screening programs among rural, largely unscreened populations.

\section{Introduction}

Effective and widespread cervical cancer screening has greatly reduced cervical cancer incidence and related morbidity and mortality ${ }^{1}$. The most commonly used cervical cancer screening method worldwide is a Pap test, which involves the collection of cervical cells for examination under a microscope by a cytopathologist. Pap testing detects abnormal cells in the cervix and enables early detection and treatment of cervical cancer ${ }^{1}$. However, Pap screening programs have low feasibility in limited-resource settings owing to a lack of infrastructure and trained personnel, limited health budgets and competing healthcare priorities ${ }^{2,3}$. To address these barriers, some national screening programs use alternatives to traditional cytology (Pap testing), such as visual inspection of the cervix with acetic acid (VIA). VIA involves unaided (naked eye) inspection of the cervix after application of acetic acid to identify abnormal tissue. While VIA eliminates some constraints of Pap testing, such as cost and need for multiple visits, there can be high variability by provider in the quality of VIA screening ${ }^{4-6}$.
DNA testing for human papillomavirus (HPV) offers an accurate alternative to VIA. The WHO recommends hr-HPV DNA testing as the primary cervical cancer screening approach in places where Pap testing has not been established ${ }^{7}$. Similar to other screening methods, cervical samples are typically gathered by a clinician during the course of a pelvic examination, but samples can also be self-collected by women themselves with a swab. WHO recommends screening with an HPV test and treatment over screening with VIA and treatment where feasible ${ }^{7}$ HPV testing is also recommended as first line screening followed by VIA and treatment ${ }^{7}$.

When successfully introduced, self-collection of samples for HPV testing can increase screening for hard to reach women or women who do not come in for screening tests ${ }^{8-11}$. Selfcollected samples have been shown to perform comparably to clinician-collected samples, but published findings suggest that the population and method of collection or testing are important to consider when assessing the utility of selfcollected samples ${ }^{12-15}$. 
would be willing to self-collect a sample at home, yet no wearch has examined self-collection in a clinical setting or whether women's hypothetical willingness would translate into actual behavior if offered an opportunity to provide a self-collected sample. We sought to assess the validity, feasibility and acceptability of using the GeneXpert HPV Assay to test self-collected vaginal samples in a rural clinic in Lilongwe District, Malawi.

\section{Methods}

\section{Study setting and population}

Women were recruited for this study as part of a larger, clinic-based study examining sexual and reproductive tract infections. Briefly, from January to August 2015, any woman who presented to the study clinic in rural Lilongwe District, Malawi, with any genitourinary symptom (including abnormal menstrual cycle or patterns of bleeding; pain with urination, pain during sex, abdominal pain, lower back pain, or any type of pelvic pain; incontinence or unusual urine odour, frequency or colour; unusual vaginal discharge in terms of quantity, odour, colour or consistency) was referred to study staff to be assessed for eligibility. Women were eligible to participate if they were 18-49 years of age, spoke Chichewa, had at least one genitourinary symptom, consented to be examined and give biological specimens for testing, and resided in Lilongwe District. Women who were pregnant or menstruating were ineligible. Women provided or thumbprint

\section{Data collection}

\section{Screening}

Women were examined in a private clinic room by the study clinician. At the start of the exam, each woman was offered the option to self-collect a vaginal sample for HPV testing.
If she agreed, she was given a sterile, cotton-tipped swab and instructions on how to collect the vaginal sample. The clinician remained in the study room, on the other side of a privacy screen, in case the participant had any questions placed the swab in $20 \mathrm{ml}$ of Preservcyt solution (Hologic, Bedford, Massachusetts) and proceeded to perform a pelvic examination. The clinician used an endocervical brush to collect the sample for HPV testing. Following collection, he swirled the cervical brush in $20 \mathrm{ml}$ of Preservcyt solution. between 2 and $8^{\circ} \mathrm{C}$ and were tested using the GeneXper HPV assay at the end of the study (Cepheid, Sunnyvale, California)

The GeneXpert technology was developed to identify multidrug resistant tuberculosis, and WHO supported the roll-out of GeneXpert systems for tuberculosis control programs in 21 countries throughout Africa and Asia ${ }^{15}$. While HPV testing using the GeneXpert is a new application of this technology, the GeneXpert platform is ubiquitous throughout Africa,
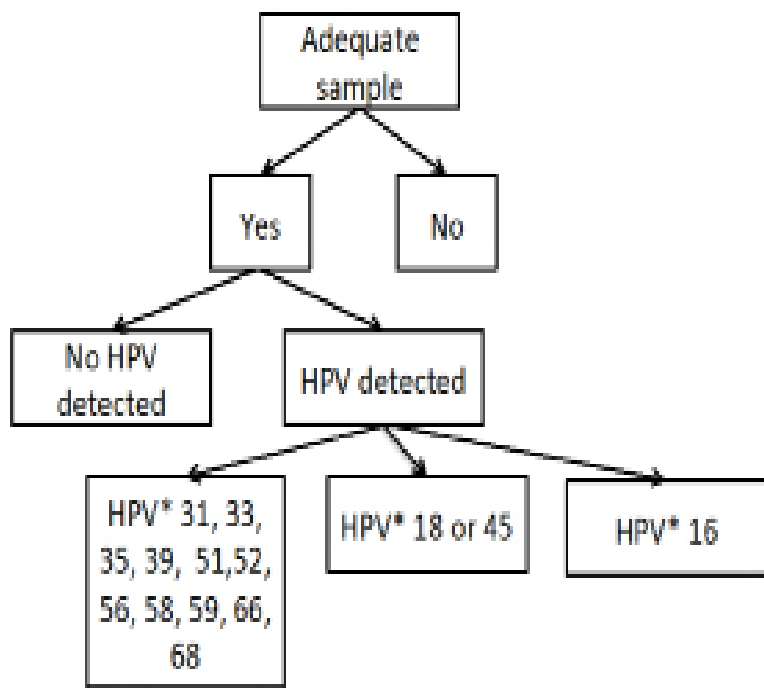

Samples will get a positive/negative result for each of the three utcomes

\section{Figure 1: Potential outcomes of GeneXpert HPV assay}

All results from the HPV testing were recorded on pape forms and ent

Questionnaire

After the clinical exam, the patient was sent to a separate study room where a research assistant, who did not provid clinical care, almistered a bilef questionnaire captuing demographic characteristics and the acceptability of the sel collection procedure. The questionnaire included items about the ease of collecting samples and understanding instructions, using a 5-point scale ranging from very easy to very difficult. using the same scale, we also assessed women's confidence in their ability to self-collect a sample, their preferences for collection of samples for HPV testing, whether they would recommend self-collection to a friend, and concerns about self-collection. Questions were developed based on previous therature and work of the study authors. All survey question were recorded directly into the Magpi data collection system (Magpi, Washington, DC) and uploaded to an internet-based storage system daily.

Testing of clinician- and self-collected samples was not completed until the end of the research study; therefore, we did not inform women if they had hr-HPV. However, we referred to VIA, and all participants in the research study underwent VIA as part f the reseatch protocol. Treatment for abnormal lesions was not available at the study clinic, and thus women with a results were referred to secondary care at one of two district hospitals in Lilongwe. The referral appointments were scheduled within a week of the study visit, and participants were provided with funds to facilitate travel to and from Lilongwe. Study clinglans requested records documenting care received at the district hospitals.
We first described the prevalence of hr-HPV in the study population, overall and by the four separate GeneXpert result categories for both self- and clinician-collected samples. We then calculated the sensitivity and specificity of selfcollected samples, using the clinician-collected samples as a reference standard. In order to assess agreement between the two sampling methods, we calculated a kappa statistic for overall hr-HPV type (positive for any hr-HPV vs. hr-HPV 16, HPV 18/45, additional hr-HPV types). As HPV testin is recommended only in women over 30 years of age due to the transient nature of HPV infections in younger women, we also conducted analyses restricting our sample to women over 30 years $(\mathrm{n}=126)$. Finally, using questionnaire data, we calculated frequencies to describe acceptability of selfcollection among participants. All analyses were done using Stata 14.0 (StataCorp, College Station, TX).

\section{Ethical approval}

This project received ethical approval from the Ohio State University Institutional Review Board and the University of Malawi College of Medicine Research and Ethics Committe

\section{Results}

\section{Study population and prevalence of $\mathrm{hr}-\mathrm{HPV}$ infections with clinician-collected sampling}

We screened 234 women to enroll 200 in the parent study, 199 of whom consented to HPV testing. Women withou hr-HPV were slightly older than women with any hr-HPV (median age 34 vs. 32 years) and more likely to be married $(97 \%$ vs. $77 \%$; Table 1$)$. Sixteen women ( $8 \%$ ) were initially noted as having a positive VIA screen although two of these were later determined to be false positives. Of the remaining 14 women, 10 presented with acetowhite lesions and four with inconclusive results that required additiona screening. All 14 women were referred to the district hospital

Table

\begin{tabular}{|c|c|c|c|c|}
\hline & $\begin{array}{c}\text { Any hr- } \\
\mathrm{n}\end{array}$ & $\begin{array}{l}-\mathrm{HPV}^{1} \\
(\%)\end{array}$ & $\begin{array}{c}\text { No hr } \\
n\end{array}$ & $\begin{array}{r}-\mathrm{HPV} \\
(\%)\end{array}$ \\
\hline Education & & & & \\
\hline & $\begin{array}{r}4 \\
10\end{array}$ & (23) & 20 & (II) \\
\hline $\begin{array}{l}2-4 \text { years } \\
5-8 \text { years }\end{array}$ & 16 & (37) & 66 & (42) \\
\hline $\begin{array}{l}5-8 \text { years } \\
>8 \text { years }\end{array}$ & 13 & (30) & 53 & (34) \\
\hline \multicolumn{5}{|l|}{ Marital status } \\
\hline Married & 33 & (77) & 151 & (97) \\
\hline Single & 10 & (23) & 5 & (2) \\
\hline \multicolumn{5}{|l|}{ Condom use last sex } \\
\hline Yes & 5 & (23) & 13 & (14) \\
\hline \multirow{2}{*}{\multicolumn{5}{|c|}{ Ever heard of cervical cancer }} \\
\hline & & & & \\
\hline Yes & 36 & (86) & 151 & (97) \\
\hline \multirow{2}{*}{\multicolumn{5}{|c|}{ Abnormal VIA }} \\
\hline & & & & \\
\hline \multirow[t]{2}{*}{$\begin{array}{l}\text { Yes } \\
\text { No }\end{array}$} & $\begin{array}{r}4 \\
39\end{array}$ & $\begin{array}{l}\text { (9) } \\
\text { (91) }\end{array}$ & $\begin{array}{r}10 \\
145\end{array}$ & (94) \\
\hline & Median & (IQR) & Median & (IQR) \\
\hline ono & $\begin{array}{c}32 \\
2\end{array}$ & $(27,36)$ & $\begin{array}{r}34 \\
2\end{array}$ & $\begin{array}{l}(29,39 \\
(12)\end{array}$ \\
\hline
\end{tabular}
for additional screening and treatment as services were not Among the women tested for HPV, 22\% ( $\mathrm{n}=43)$ had any wpe of hr-HPV by clinician-collected sample (Table 2). Six

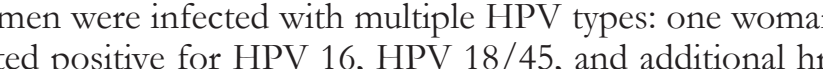
HPV type(s), 4 women had both HPV 18/45 and additional hr-HPV type(s), and one woman had HPV 16 and additiona hr-HPV type(s).

\section{Agreement between clinician-collected and self-} ected samples

We found high agreement between HPV results from selfand clinician-collected samples as measured by the kappa statistic, which measures agreement beyond chance alone. samples was for HPV 18/45 $(x=0$. 90). The agreement between self- and clinician-collected samples for any type of hr-HPV and the additional hr-HPV types were similar collected $x=0.74$, Table 3 ). Overall compared to clinicianspecific and varied in sensitivity by type of HPV (Tible 3 ) specific and varied in sensitivity by type of HPV (Table 3). type, sensitivity was poorer; we found the highest sensitivity

\section{Table 2: HPV results, by collection modality}

\begin{tabular}{lrrrl}
\hline & \multicolumn{2}{c}{ Clinician } & \multicolumn{1}{c}{ Self } \\
\hline & & & & \\
& $\mathrm{n}$ & $(\%)$ & $\mathrm{n}$ & $(\%)$ \\
\cline { 2 - 5 } None $^{1}$ & 156 & $(78)$ & 166 & $(83)$ \\
Any hr-HPV & 43 & $(22)$ & 33 & $(17)$ \\
HPV 16 & 8 & $(4)$ & 4 & $(2)$ \\
HPV 18/45 & 11 & $(6)$ & 11 & $(6)$ \\
Additional hr-HPV & 23 & $(15)$ & 24 & $(12)$ \\
\hline
\end{tabular}

Additional hr-HPV

$23 \quad(15$

for HPV 18/45 at 91\% (95\% CI: 59\%, 100\%) and lowest sensitivity for HPV 16 at $50 \%$ (95\% CI: $16 \%, 84 \%)$.

When restricting the analyses to women older than 30 years, based on current that there was increased sensitivity for
thating detection of all hr-HPV types combined, HPV 16 and additional hr-HPV, but a decrease in sensitivity for detection of HPV 18/45 (Table 4). The exclusion of younger women also led to an increase in most kappa values characterizing greement between clinician and selfcollected samples, including for a $16(x=0.85)$ and the additional hr-HPV category $(x=0.77)$. Restricting to older women (who are likely to have fewer transient infections) we found that in general there was increased kappa agreement and sensitivity although it was not consistent across all hr-HPV types.

\section{Acceptability}

Overall, women found the selfcollection procedure easy to perform $(98 \%)$, reported that confident they did it correctly ( $95 \%$; Table 5). Most women 
Table 4: Concordance between HPV test results by self- and clinician-collected samples in

women older than 30 years of age

\begin{tabular}{lrlrrrr} 
& Sensitivity & $(95 \% \mathrm{CI})$ & Specificity & $(95 \% \mathrm{CI})$ & Kappa $^{1}$ & $(95 \% \mathrm{CI})$ \\
\hline All types of hr- HPV & 79 & $(57,93)$ & 99 & $(95,100)$ & 0.83 & $(0.71,0.96)$ \\
HPV 16 & 75 & $(19,99)$ & 100 & $(97,100)$ & 0.85 & $(0.57,1.0)$ \\
HPV 18/45 & 83 & $(36,100)$ & 99 & $(95,100)$ & 0.82 & $(0.59,1.0)$ \\
Other hr-HPV & 75 & $(48,93)$ & 98 & $(94,100)$ & 0.77 & $(0.60,0.95)$
\end{tabular}

\section{Kappa measures expected vs, observed agreement}

\section{Table 5: Acceptability of self-collection ${ }^{1}$}

\begin{tabular}{lll}
\hline & $\mathrm{n}$ & $(\%)$ \\
\hline Very or somewhat easy to understand the self-collection instructions & 197 & $(100)$ \\
Recommend self-collection to a friend & 193 & $(99)$ \\
Very or somewhat easy to do self-collection of samples & 194 & $(98)$ \\
Would use self-collection for HPV testing in the future & 194 & $(99)$ \\
Very or somewhat certain self-collected correctly & 186 & $(95)$ \\
Prefer self-collection over clinician-collection & 119 & $(61)$ \\
\hline Concerns about self-collection & 138 & \\
\hline No concerns & 22 & $(12)$ \\
Might hurt & 20 & $(11)$ \\
Might not be accurate & 14 & $(7)$ \\
Might not do it correctly & &
\end{tabular}

Might not do it correctly

'Women could select multiple concerns
reported they would recommend self-collection for HPV other tests from a range of settings (kappa values ranging testing to a friend $(99 \%)$ and more women preferred self- from $0.70-0.87)^{20-24}$. Our findings also align with previou collection compared to clinician-collection sampling $(61 \%$ vs. $39 \%$ ).

Three-quarters of women $(74 \%)$ reported they would not have any concerns about self-collecting for HPV testing reported worries that self-swabbing might hurt, $11 \%$ feared the HPV results following self-collection might not be accurate, and $7 \%$ were concerned they may not test correctly.

\section{Discussion}

To our knowledge, this is the first study to examine selfcollected samples for HPV testing using the GeneXpert HPV assay in a low resource setting. Our findings sugges that self-collection of samples for HPV testing is a feasible and acceptable method of cervical cancer screening in this rural, Malawian population. While the specificity was high, we found lower levels of sensitivity suggesting that selfcollected samples for HPV testing may not be as valid as clinician-collected samples. The self-collection procedure was easy to incorporate into the clinic setting with all but one participant providing a sample, and all collected samples were sufficient for testing. High agreement between the self- and clinician-collected samples, and high levels of acceptability among participants, suggest that self-collection procedures for HPV testing may be effectively incorporated into screening programs among rural, largely unscreened populations in Malaw

but high agreement in HPV test results between self- an research that suggests agreement in HPV results from selfand clinician-collected samples can vary by HPV type While the specificity of the self-collected tests was very high in our study, sensitivity varied by type of HPV. In othe words, HPV testing using self-collected samples accurately detected HPV-negative women, but the ability to detec HPV-positive women using self-collected samples was more variable. When younger women were excluded from the sample, sensitivity was more comparable across the differen types of hr-HPV, although the sensitivity for detection of HPV 18/45 was slightly reduced. This overall pattern of lower sensitivity but higher specificity of self-collected vs. clinician-collected samples is similar to findings from a study of Gambian women, where self-collected samples had sensitivity of 0.64 ( $5 \%$ CI: $0.52,0.83)$ and specificity of 0.94 compared to clinician-collected cervical samples

Our findings suggest that self-collection of samples for HPV testing was widely acceptable, easy to perform and preferred to clinician-collection. Combined with results from other research, our study provides evidence that self-collection could be used in an outreach capacity to increase screening in hard-to-reach populations. For example, among women in Argentina, women who were offered the opportunity to self-collect a sample in the home through community healt workers were four times as likely to be screened for cervical cancer than women who were not offered the option to selfcollect ${ }^{15}$. In a randomized control trial conducted in Uganda $98 \%$ of women in the HPV self-collection arm were screened for cervical cancer while in the control arm (VIA), only $48 \%$ of women received cervical cancer screening ${ }^{2}$.
To be successfully implemented, screening programs using way. While we did not deal with the whole scope of HPV testing will need to consider clinician and laboratory necessary care, we did assess one part of the pathway, and perspectives alongside other programmatic considerations. For example, in our project, the study clinician found that after a small number of participants had enrolled, it was very simple to collect cervical samples using the cervical brush. On the other hand, he experienced challenges explaining the self-collection procedure to women, suggesting that a future screening program must provide detailed instructions or have present a healthcare provider to answer questions. The laboratory technician found it easy and fast to test samples using the GeneXpert HPV test. However, some samples required a repeat test due to machine error and added to costs of HPV testing. We also experienced challenges in procuring the transport medium - an expensive part of the testing procedure, and this supply issue must be addressed before a larger rollout of any HPV testing program is possible in this region. Studies of other polymerase chain reaction (PCR)-based HPV DNA tests suggest that more commercially-available and inexpensive transport media (e.g. Scope mouthwash ${ }^{27}$ ) or the collection and storage of dry samples, may perform comparably ${ }^{27,28}$, although to date these approaches have not been validated for the GeneXpert HPV DNA test.

Our findings must be interpreted in light of important stud limitations. As our project was undertaken as a secondar arm of a larger study, the population included younger women (under age 30) for whom HPV testing is not currently recommended. However, subgroup analyses with older women in the recommended range suggest that kappa of self- and clinician-collected samples and sensitivity and specificity findings were valid for both groups. As this project was nested in a pilot study, we had a relatively small sample size, especially when excluding women under age 30 . The smaller sample size may have influenced the lower sensitivity for self-collected samples. Generalizability of our results may also be limited as we enrolled care-seeking women who presented with genitourinary symptoms to a medical facility. As HPV infections and early cervical lesions do not lead to noticeable symptoms, it will be important to determine whether HPV screening (via self-collected samples) remains acceptable in women without genitourinary symptoms. We also enrolled women presenting at a clinic, so we cannot extrapolate our results to cervical cancer screening programs in an outreach capacity. Additionally, our study clinician was in the room during self-collection of the sample which may not be generalizable to an outreach setting where there are not trained personnel available. Our study also only assessed the feasibility of self-collection compared to clinician-collected sampling, but we note that $\mathrm{WHO}$ guidelines recommend immediate treatment be available for any clinic-based cervical cancer screening program. Lastly, the acceptability questions may be influenced by response bias with participants voicing more favorable views on self-collection than they actually felt. We attempted to minimize this bias by having a nonclinician research assistant deliver the questions in a separate, tudy room

While the rates of cervical cancer incidence and mortality have decreased precipitously in the last 40 years globally, the burden of disease falls disproportionally on women in low-resource settings without accessible screening programs. Although we have demonstrated some of the utility of screening, Malawians remain with significant challenges in
providing appropriate therapy in a reliable and sustainable demonstrated that it could be useful in the arc of care that women need.

\section{Conclusion}

Self-collecting samples for HPV testing has the potential to eliminate many of the barriers of other cervical cance resource settings. We submit that cervical cancer-screening programs using self-collected samples for HPV testing may
be a feasible, valid, acceptable, and effective cervical cancer screening method in this rural Malawian population.

\section{Acknowledgements}

The project was supported by Award Number Grant TL1TR001069 from the National Center For Advancing Translational Sciences. The content is solely the responsibility of the authors and does not necessarily represent the official views of the National Center For Advancing Translational Sciences or the National Institutes of Health.

\section{References}

1. International Agency for Research on Cancer. Cervix cancer screening. Lyon; 2005 .

2. Campos NG, Kim JJ, Castle PE, Ortendahl JD, O'Shea M, Diaz M, et . Health and economic impact of HPV 16/18 vaccination and cervical doi: $10.1002 /$ ijc. 26269

3. Khozaim K, Orang'o E, Christoffersen-Deb A, Itsura P, Oguda J, uliro $\mathrm{H}$, et al. Successes and challenges of establishing a cervical Gynaecol Obstet Off Organ Int Fed Gynaecol Obstet 2013;124:12-8. doi:10.1016/j.ijgo.2013.06.035. doi 10,1016/j.ijgo.2013.06;.035

4. Ajenifuja K, Gage J. A population-based study of visual inspection with acetic acid (VIA) for cervical screening in rural Nigeria. Int 5. Longatto-Filho A, Naud P, Derchain SF, Roteli-Martins C, Tatti S, Hammes LS, et al. Performance characteristics of Pap test, VIA, ILI, HR-HPV testing, cervicography, and colposcopy in diagnosis

6. Vet JNI, Kooijman JL, Henderson FC, Aziz FM, Purwoto G, Susanto H, et al. Single-visit approach of cervical cancer screening: see and treat . B J Cancer 2012,107.72-7. doi.10.1038/jc.201233 . World Health Organization. WHO guidelines for screening and 8. Racey CS, Withrow DR, Gesink D. Self-collected HPV testing mproves participation in cervical cancer screening: a systematic review and meta-nnalysis. Can J Public Health 2013:104:e159-66.

9. Vanderpool RC, Jones MG, Stradtman LR, Smith JS, Crosby RA. Selfcollecting a cervico-vaginal specimen for cervical cancer screening women in rural appalachia. Gynecol Oncol 2013:132.521-5, do: 10.1016/j.ygyno.2013.10.008. doi: 10.1016/j.ygyno.2013.10.008

10. Ogilvie G, Krajden M. Feasibility of self-collection of specimens for human papillomavirus testing in hard-to-reach women. CMAJ 11. Gök M, van Kemenade FJ, Heideman D A, Berkhof J, Rozendaal testing on vaginal brush-based self-samples of non papillomaviru $\frac{\text { testing on vaginal brush-based self-samples of non-attendees of the }}{\text { https: } / / d x . d o i . o r g / 10.4314 / \mathrm{mmj} . v 30 i 2.2}$ screening programs that restrict access for women in low-

\section{Conflict of interest} Gynecol Cancer 2013;23:507-12. doi: 10.1097/IGC.0b013e318280f39 of significant cervical pathology. Virchows Arch
2012;460:577-85. doi: 10.1007/s00428-012-1242-y 
cervical screening program. Int J Cancer J Int Cancer 2012;130:112835. doi: $10.1002 /$ ijc. 26128 .

12. Arriba LN, Enerson CL, Belinson S, Novick L, Belinson J. Mexican Cervical Cancer Screening Study II: acceptability of human papillomavirus self-sampler. Int J Gynecol Cancer Off J Int Gynecol Cancer Soc 2010;20:1415-23. doi: 10.1111/IGC.0b013e3181f58678

13. Mitchell S, Ogilvie G, Steinberg M, Sekikubo M, Biryabarema C, Money D. Assessing women's willingness to collect their own cervical samples for HPV testing as part of the ASPIRE cervical cancer screening project in Uganda. Int $\mathrm{J}$ Gynaecol Obstet Off Organ Int Fed Gynaecol Obstet 2011;114:111-5. doi: 10.1016/j.ijgo.2011.01.028

14. Kahn JA, Bernstein DI, Rosenthal SL, Huang B, Kollar LM, Colyer JL, et al. Acceptability of human papillomavirus self testing in female adolescents. Sex Transm Infect 2005;81:408-14. doi: 10.1136/ sti.2004.012047.

15. Arrossi S, Thouyaret L, Herrero R, Campanera A, Magdaleno A, Cuberli M, et al. Effect of self-collection of HPV DNA offered by community health workers at home visits on uptake of screening for cervical cancer (the EMA study): a population-based clusterrandomised trial. Lancet Glob Health 2015;3:e85-94. doi: 10.1016/ S2214-109X(14)70354-7.

16. Rossi PG, Marsili LM, Camilloni L, Iossa A, Lattanzi A, Sani $\mathrm{C}$, et al. The effect of self-sampled HPV testing on participation to cervical cancer screening in Italy: a randomised controlled trial (ISRCTN96071600). Br J Cancer 2011;104:248-54. doi: 10.1038/ sj.bjc. 6606040 .

17. Sanner K, Wikstrom I, Strand A, Lindell M, Wilander E. Selfsampling of the vaginal fluid at home combined with high-risk HPV testing. Br J Cancer 2009;101:871-4. doi: 10.1038/sj.bjc.6605194.

18. Urasa M, Darj E. Knowledge of cervical cancer and screening practices of nurses at a regional hospital in Tanzania. Afr Health Sci 2011;11:48-57.

19. Snijders PJF, Verhoef VMJ, Arbyn M, Ogilvie G, Minozzi S, Banzi R, et al. High-risk HPV testing on self-sampled versus cliniciancollected specimens: a review on the clinical accuracy and impact on population attendance in cervical cancer screening. Int J Cancer J Int
Cancer 2013;132:2223-36. doi: 10.1002/ijc.27790

20. Jones HE, Brudney K, Sawo DJ, Lantigua R, Westhoff CL. The acceptability of a self-lavaging device compared to pelvic examination for cervical cancer screening among low-income women. J Womens Health 2012;21:1275-81. doi: 10.1089/jwh.2012.3512

21. Adler DH, Almudevar A, Gray GE, Allan B, Williamson A-L. High level of agreement between clinician-collected and self-collected samples for HPV detection among South African adolescents. J Pediatr Adolesc Gynecol 2012;25:280-1. doi: 10.1016/j.jpag.2012.04.006

22. Safaeian M, Kiddugavu M, Gravitt PE, Ssekasanvu J, Murokora D, Sklar M, et al. Comparability of self-collected vaginal swabs and physician-collected cervical swabs for detection of human papillomavirus infections in Rakai, Uganda. Sex Transm Dis 2007;34:429-36. doi: 10.1097/01.olq.0000243623.67673.22

23. Holanda F, Castelo A, Veras TMCW, de Almeida FML, Lins MZ, Dores GB. Primary screening for cervical cancer through self sampling. Int J Gynaecol Obstet Off Organ Int Fed Gynaecol Obstet 2006;95:17984. doi: 10.1016/j.ijgo.2006.07.012

24. Schmeink CE, Bekkers RLM, Massuger LF a G, Melchers WJG. The potential role of self-sampling for high-risk human papillomavirus detection in cervical cancer screening. Rev Med Virol 2011;21:139-53. doi: 10.1136/sti.2004.010413.

25. Lack N, West B, Jeffries D, Ekpo G, Morison L, Soutter WP, et al. Comparison of non-invasive sampling methods for detection of HPV in rural African women. Sex Transm Infect 2005;81:239-41. http://dx.doi. org/10.1136/sti.2004.010413

26. Moses E, Pedersen HN, Mitchell SM, Sekikubo M, Mwesigwa D, Singer J, et al. Uptake of community-based, self-collected HPV testing vs. visual inspection with acetic acid for cervical cancer screening in Kampala, Uganda: Preliminary results of a randomized controlled trial. Trop Med Int Health 2015;20:1355-67. doi: 10.1111/tmi.12549

27. Gage JC, Partridge EE, Rausa A, Gravitt PE, Wacholder S, Schiffman M, et al. Comparative performance of human papillomavirus DNA testing using novel sample collection methods. J Clin Microbiol 2011;49:4185-9. doi: 10.1128/JCM.01254-11.

28. Eperon I, Vassilakos P, Navarria I, Menoud P-A, Gauthier A, Pache J-C, et al. Randomized comparison of vaginal self-sampling by standard vs. dry swabs for human papillomavirus testing. BMC Cancer 2013;13:353. doi: 10.1186/1471-2407-13-353. 